\title{
Research of Theoretical Model and Transmission Efficiency of ZC1 Worm Dynamic Lubrication
}

\author{
Dongyue $\mathrm{Qu}^{\mathrm{a}}$, Chunyu Zhang ${ }^{\mathrm{b}}$, Jianan $\mathrm{Xu}^{\mathrm{c}}$, Rilai Zhang and \\ Shengxi Zhang \\ Harbin Engineering University, Harbin 150001, China \\ aqudongyue@hrbeu.edu.cn, b18944601951@163.com, ‘xujianan@hrbeu.edu.cn
}

Keywords: Circular cylindrical worm, Instantaneous contact point, Induced curvature, Dynamic pressure lubrication model, Transmission efficiency

Abstract. The dynamic pressure lubrication theoretical model of worm gear ZC1 is established, the formula of hydrodynamic lubrication discretization for the calculation of the mixed friction efficiency of circular cylindrical worm and worm pair is achieved and the formula and process for solving the hybrid friction efficiency according to the model which is given. Through the calculation of specific examples, the transmission efficiency was calculated to be $84.25 \%$ at a worm angle of $\varphi 1=90^{\circ}$. The establishment of the discretization model of hydrodynamic lubrication of circular cylindrical worm and the calculation of its efficiency are of great significance for the lubrication research and the design of basic parameters.

\section{Introduction}

Since the middle of the last century, the Neiman worm has been proposed, this type of circular cylindrical worm gear has attracted the attention of many scholars because of its lubrication angle is larger and transmission quality is superior to ordinary worm drive [1]. Prof. B.-R. Höhn [2], Technical University of Munich, Germany, aimed for making the improvement on the quality of the circular cylindrical worm drive, selected the meshing position and the meshing area of the worm gear as the objective function to optimize the transmission quality. In addition, Professor Wang Shuren and his team [3], Tianjin University, China, studied the formation of circular toroid enveloping cylindrical worm gear tooth surface, the area of the contact area was also calculated, and the transmission quality was optimized by optimizing the contact area.

At present, the optimization research on the transmission efficiency and meshing quality of circular cylindrical worm gear pairs has not considered the influence of surface roughness, lubricating oil and viscosity changes to the efficiency, and doesn, $t$ consider the meshing condition of the entire instantaneous contact line. Therefore, in this paper, based on the previous research results, the circular toroidal helical worm pair with toroidal envelope is discretized and a dynamic pressure lubrication model is established. A theoretical model of hybrid friction lubrication with circular cylindrical worm gear is presented. The solving process of transmission efficiency is given, which plays an active role in the research of hybrid friction theory on the optimization of arc cylindrical worm pairs.

\section{Curvature Calculation of Instant Engagement Point of ZC1 Worm Drive}

\subsection{Instantaneous Contact Line of ZC1 Worm Pair.}

Taking $\mathrm{z} 1=2$ circular cylindrical worm gear pair as an example to draw the instantaneous mesh line shown in Fig. 1. It can be seen from the distribution of the meshing line that the shape of the engagement line of the circular cylindrical worm pair is more favorable to the formation of the hydrodynamic oil film than that of the ordinary worm pair. As the worm angle $\varphi 1$ increases from $-135^{\circ}$ to $180^{\circ}$, the lubrication angle increases at each point of the contact line, but there are two instantaneous contact lines at $\varphi 1$ of $-135^{\circ}$ and $180^{\circ}$. In addition, in the meshing exit area of the instantaneous contact line, the contact point lubrication angles are larger than that in other areas, and 
the shape of contact line is more conducive to the formation of dynamic pressure oil film, and the lubrication conditions is better.

\subsection{Curvature Solution.}

Equation (1) is the formula of the normal curvature $k_{n}^{(1)}$ at the meshing point of the worm gear[4], and equation (2) is the formula of the induction curvature along the normal direction of the instantaneous contact line.

$$
\begin{aligned}
& k_{n}^{(1)}=H+R \cos \left(\frac{2 \mu}{\lambda}\right) \\
& H=\frac{\left(k_{1}+k_{2}\right)}{2}=\frac{E N-2 F M+G L}{2\left(E G-F^{2}\right)} \\
& R=\frac{\left(k_{1}-k_{2}\right)}{2}=\frac{\sqrt{(E N-2 M+G L)^{2}+4(E G-F)^{2}(L N-M)^{2}}}{2\left(E G-F^{2}\right)} \\
& k_{n}^{(12)}=\frac{(\vec{N})^{2}}{\psi}
\end{aligned}
$$

The curvature at the meshing point of the worm wheel can be obtained by the calculation flow which is shown in Figure 2.

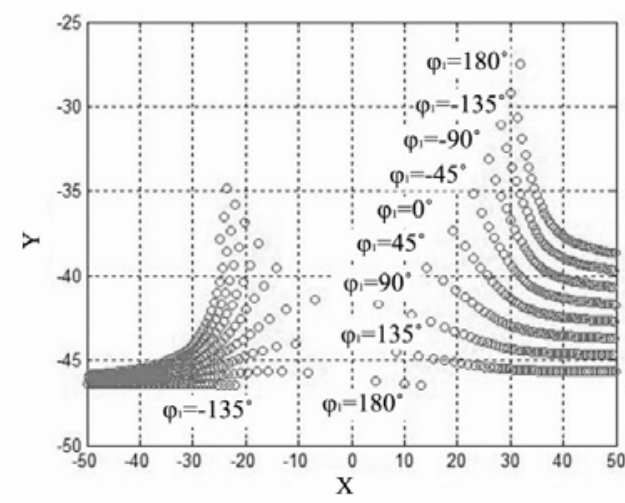

Fig. 1 Worm gear momentary contact diagram

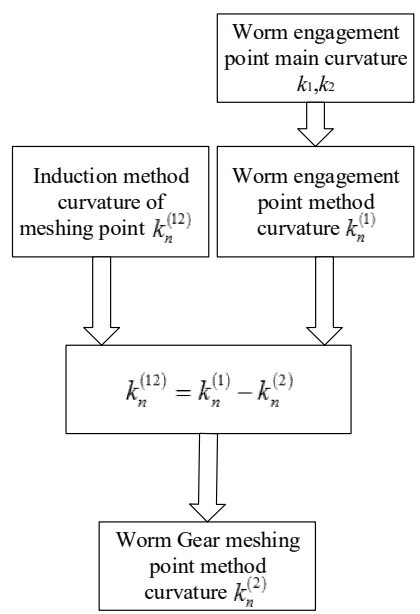

Fig. 2 Turbine meshing point method curvature calculation flow

\section{Dynamic Pressure Lubrication Model Establishment and Efficiency Calculation}

\subsection{Discretization Model.}

At any contact point of the meshing line, the tooth surfaces of the worm and the worm gear can be approximated as two cylinders, and the dynamic pressure lubrication discretization model is as shown in Fig. 3. However, the circular cylindrical worm gear is a concave-convex fit, the schematic diagram of the hydrodynamic lubrication discretization model shown in Fig. 4.

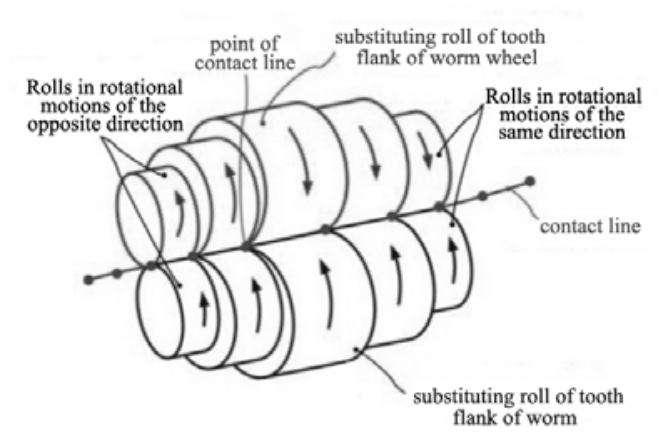

Fig.3 Ordinary worm dynamic pressure lubrication discretization model

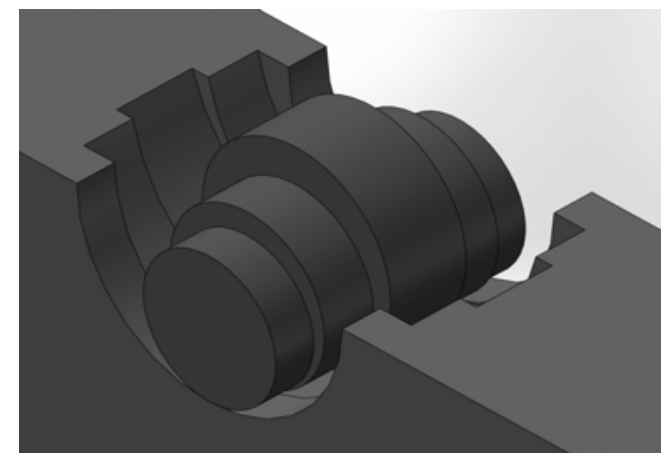

Fig.4 Discrete model of circular cylinder worm dynamic pressure lubrication 


\subsection{Calculation and Process of Transmission Efficiency.}

Assuming that the height of surface asperities conforms to Gaussian distribution, the probability density function can be calculated by Equation (3)[5].

$$
\varphi\left(h_{s}\right)=\frac{1}{\sqrt{2 \cdot \pi}} \cdot \exp \left(-\frac{1}{2} \cdot\left(\frac{h_{s}-\bar{h}_{s}}{\sigma_{s}}\right)^{2}\right)
$$

According to the above description, the calculation procedure of instantaneous transmission efficiency of worm pair is shown in Fig. 5.

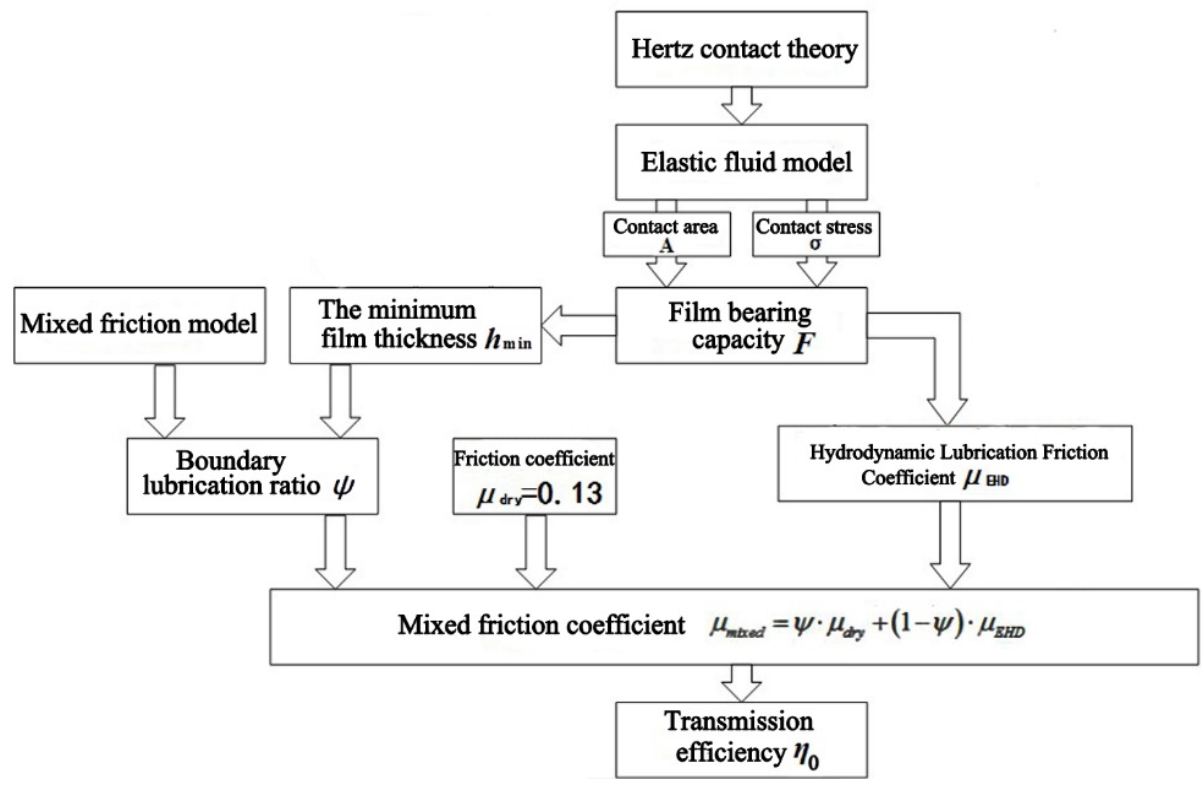

Fig. 5 Circular cylindrical worm drive efficiency of the flow chart

\section{Example and Result Analysis}

In this paper, arc cylindrical worm gear optimization example parameters are as follows: $a=250 \mathrm{~mm}, \mathrm{~m}=7.4 \mathrm{~mm}, \rho=38 \mathrm{~mm}, \mathrm{zl}=2, \mathrm{z} 2=55, \alpha=23^{\circ}, \gamma=10^{\circ}, \mathrm{d} 1=84 \mathrm{~mm}, \mathrm{x}=0.608$. The relative parameters of the circular cylindrical worm gear pair are calculated by using MATLAB. When the worm angle $\varphi 1=90^{\circ}$, the instantaneous meshing line is obtained as shown in Figure 6. Part of parameters are shown in Table 1.

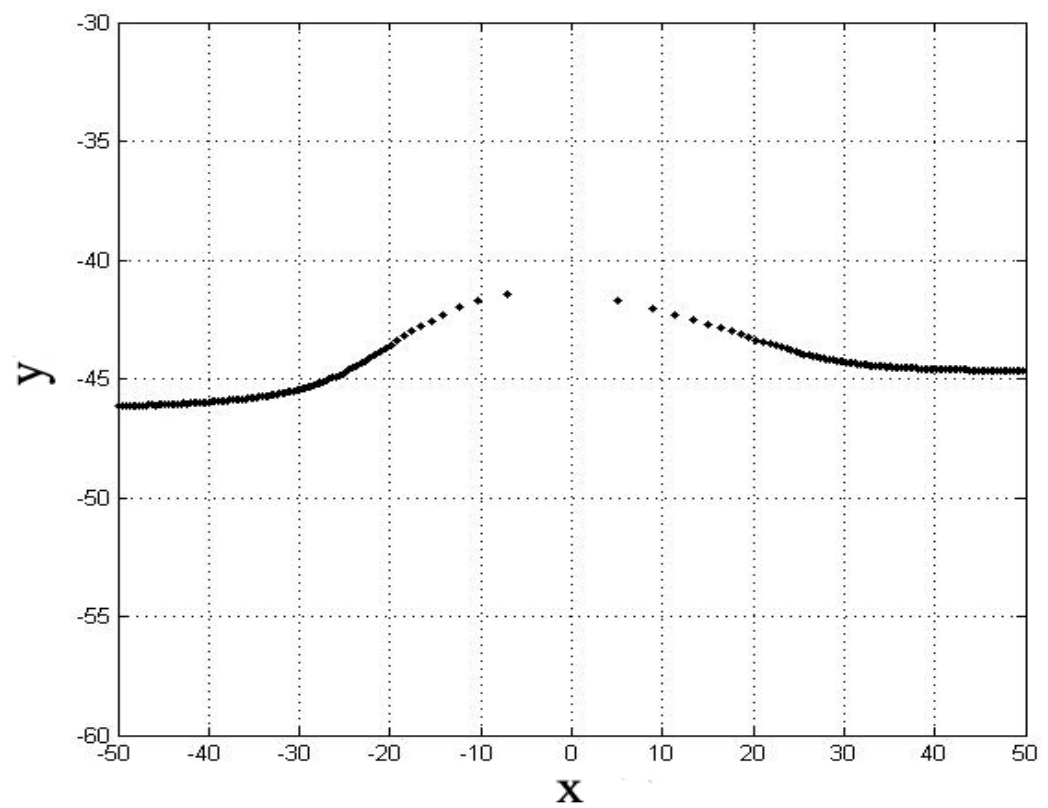

Fig.6 Worm momentary engagement at $\varphi 1=90^{\circ}$ 
Table 1 Instantaneous contact point part of the parameter list

\begin{tabular}{cccccccc}
\hline$\beta$ & $0^{\circ}$ & $1^{\circ}$ & $2^{\circ}$ & $3^{\circ}$ & $5^{\circ}$ & $7^{\circ}$ & $10^{\circ}$ \\
\hline $\mathrm{x}$ & 5.133 & 21.122 & 26.524 & 30.211 & 36.633 & 43.147 & 53.671 \\
$\mathrm{y}$ & -41.685 & -43.459 & -44.037 & -44.316 & -44.537 & -44.631 & -44.691 \\
$k_{n}^{(1)}$ & -3864.039 & -1428.538 & -2877.062 & -2856.427 & -3714.288 & -3642.720 & -3267.046 \\
$k_{n}^{(2)}$ & 3864.034 & 1428.528 & 2877.049 & 2856.446 & 3714.268 & 3642.699 & 3267.024 \\
$\theta$ & $13.112^{\circ}$ & $34.358^{\circ}$ & $37.286^{\circ}$ & $38.390^{\circ}$ & $41.373^{\circ}$ & $45.321^{\circ}$ & $51.262^{\circ}$ \\
\hline
\end{tabular}

From Figure 6 and Table 1, it can be seen that when the worm angle $\varphi 1=90^{\circ}$, the lubrication angle $\theta$ of the entrance and exit of the meshing zone is larger and the lubrication condition is better. At the middle meshing position, the lubrication angle $\theta$ is close to $0^{\circ}$ and the lubrication conditions is the worst, anti-adhesive wear ability is the worst as well.

The obtained profiles of oil film thickness and oil film pressure at each engagement point are shown in Fig. 7 and Fig. 8.

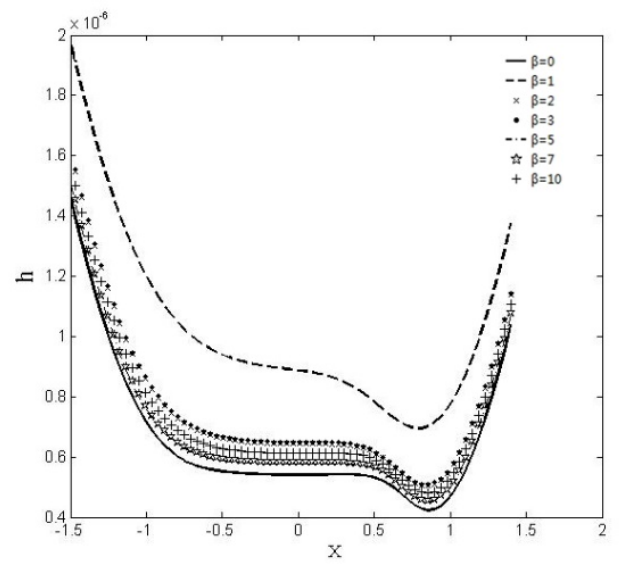

Fig.7 The figure of instantaneous meshing point when oil film distribution at $\varphi 1=90^{\circ}$

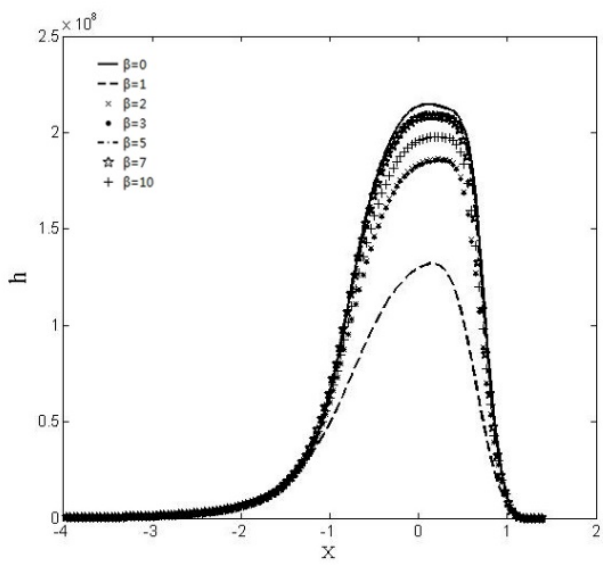

Fig. 8 The figure of instantaneous meshing point hydraulic pressure distribution at $\varphi 1=90^{\circ}$

Gaussian distribution of random surface roughness model is as shown in Figure 9.

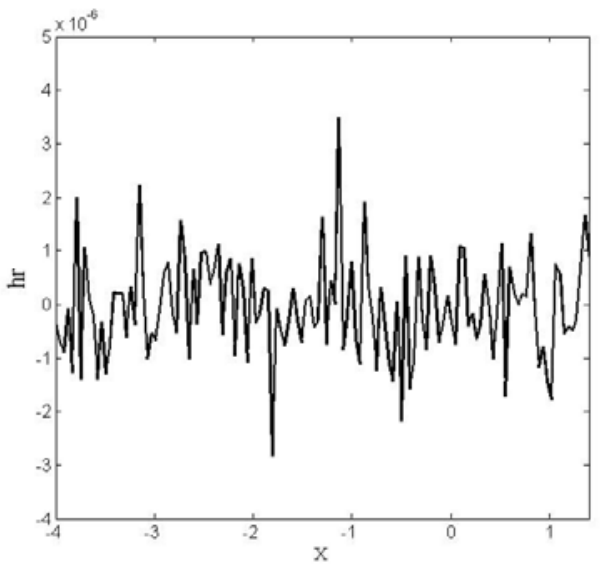

Fig. 9 Gaussian distribution random surface roughness distribution

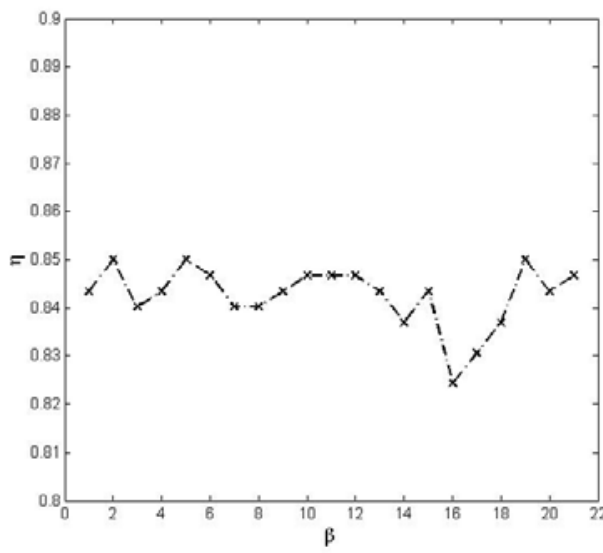

Fig. 10 Meshing point transmission efficiency curve at $\varphi 1=90^{\circ}$ 
Figure 10 is the transmission efficiency curve of all the meshing points, assuming that each meshing point has the same effect of the entire meshing line lubrication, the final instantaneous transmission efficiency can be calculated by Equation (4).

$$
\eta_{0}=\sum_{1}^{i} \eta_{i} / i=84.25 \%
$$

\section{Summary}

According to the theoretical meshing mathematical model of worm and worm gear which is derived from the theory of engagement and the principle of circular cylindrical worm gear, the instantaneous contact line trend of the worm cylinder with $z 1=2$ is obtained and the following conclusions are obtained:

(1) The distribution of the contact lines is larger at the exit of the meshing area and is slightly smaller at the entrance, and the contact line is the sparsest at the middle meshing area, indicating that the instantaneous coincidence coefficient of the exit area is the largest and the middle area is the smallest.

(2) It can be seen that the lubrication angle at the exit and entrance of the contact line is larger than that at the middle area. The lubrication point at the contact point is equal to $0^{\circ}$ at the middle area and has a bad influence on the wear of the worm.

(3) The formulas of the induced curvature and the induced curvature at the meshing point between the worm and the worm gear are deduced. According to the establishment of the circular cylindrical worm dynamic pressure lubrication discretization model, the final drive efficiency is calculated to be $84.25 \%$, which provided a good idea for the research on the efficiency of circular cylindrical worm gear pair transmission, and it also has certain research significance for the experiment.

\section{References}

[1]. Jianfeng Dong, Xiuming Weng, Kun Wang, et al. Worm gear drive technology in our new progress [J]. Mechanical Design and Manufacturing Engineering, 2014,43 (4): 78-81., in Chinese,

[2]. Höhn B R, Steingrover K, Lutz M. Determination and optimization of the contact pattern of worm gears[J]. VDI Berichte, 2002, 1(1665), 341-352.

[3]. Shuren Wang, LinFeng Chang, Mengxia Lin, Jianliang Zhang. Fuzzy Optimization Design of Escalator ZC1 Worm Gear [J]. Mechanical Design, 2001, (4): 12-14., in Chinese,

[4]. Lijuan Song, Weixi Ji, Jian Xie, et al. Analysis and optimization of contact parameters of worm gear pair ZC1 [J]. Journal of Mechanical Transmission, 2016 (4): 81-86., in Chinese,

[5]. Magyar B, Sauer B, Horak P. Tribological Investigation of K Type Worm Gear Drives[J]. Acta Polytechnica Hungarica, 2012, 9(6):233-252. 